\title{
Daya Bay energy calibration model
}

\section{Vit Vorobel on behalf of Daya Bay collaboration}

Faculty of Mathematics and Physics, Charles University, Prague

12116 Praha 2, Ke Karlovu 3, Czech Republic

E-mail: vit.vorobelemff.cuni.cz

The Daya Bay Reactor Neutrino Experiment was designed to determine $\theta_{13}$, the smallest mixing angle in the three-neutrino mixing framework, with unprecedented precision. Daya Bay provided $\theta_{13}$ with the best precision and made an independent measurement of the effective mass splitting in the electron antineutrino disappearance channel [1]. Daya Bay also performed a number of other precise measurements, such as a high-statistics determination of the absolute reactor antineutrino flux and spectrum, and study of their evolution, as well as a search for sterile neutrino mixing, among others. A precision evaluation of the antineutrino energy spectra is a fundament for neutrino oscillation investigation, so an accurate conversion of the detector response to the antineutrino energy is a key attribute of the analysis. This is named as the detector energy response model, which is a subject of the presentation. The model involves the major sources of energyresponse non-linearity which originates from processes in the liquid scintillator and readout electronics. They are treated differently for response to electrons, gamma's and positrons. Also other potential biases related to energy calibration are taken into account.

The 39th International Conference on High Energy Physics (ICHEP2018)

4-11 July, 2018

Seoul, Korea 
The detector response to electrons is reduced due to scintillation quenching which is in the model described with Birks formula

$$
f_{q}\left(E_{\text {true }}, k_{B}\right)=\int_{0}^{E_{\text {true }}} \frac{d E}{1+k_{B} \times \frac{d E}{d x}}
$$

The linear electron energy loss $d E / d x$ is evaluated using Monte Carlo (MC) simulations and the Birks coefficient $k_{B}$ is one of the model parameters.

Cerenkov radiation contribution together with the scintillation quenching are involved in the model according to the formula

$$
f_{\text {scint }}=\frac{E_{\text {vis }}}{E_{\text {true }}} \propto f_{q}\left(E_{\text {true }}, k_{B}\right)+k_{C} \cdot f_{C}\left(E_{\text {true }}\right)
$$

The ratio of Cerenkov radiation to scintillation $k_{C}$ is the model parameter and the function $f_{C}\left(E_{\text {true }}\right)$ is calculated with MC.

Electronics imperfect charge integration is calculated using the function

$$
f_{\text {elec }}=\frac{E_{\text {elec }}}{E_{\text {vis }}} \propto \alpha e^{-\frac{E_{\text {vis }}}{\tau}}+1-\alpha e^{-\frac{E_{\text {anchor }}}{\tau}}
$$

Size of electronics nonlinearity $\alpha$ and electronics-nonlinearity time constant $\tau$ are the model parameters determined from dedicated measurements. This model describing electrons is adapted to gamma's and positrons.

Gamma's convert to electrons via Compton scattering and the photo-effect. The density of probability $P\left(T_{e-}\right)$ that an electron has energy $T_{e-}$ is determined from MC. Then the energy visible with the detector can be evaluated as the integral

$$
E_{v i s}^{\gamma}=\int_{0}^{T_{\text {max }}} P\left(T_{e^{-}}\right) \times T_{e^{-}} \times f_{\text {scint }}\left(T_{e^{-}}\right) d T_{e^{-}}
$$

Positrons are assumed to behave as electrons except for annihilation to 2 gamma's when at rest. Annihilation in flight, and 3-gamma decay are neglected in the model.

$$
E_{\text {vis }}^{e^{+}}=T_{e^{+}} \times f_{\text {scint }}^{e^{-}}\left(T_{e^{+}}\right)+2 \times E_{\text {vis }}^{\gamma}(511 \mathrm{keV})
$$

Final formula for non-linearity of response to positrons including all described aspects is

$$
f_{N L}^{e^{+}}\left(T_{e^{+}}\right)=A \times \frac{E_{\text {vis }}^{e^{+}}}{E_{\text {true }}^{e^{+}}} \times f_{\text {elec }}\left(A \times E_{\text {vis }}^{e^{+}}\right)
$$

The absolute energy scale $A$ is a parameter of the model.

The five free parameters in the model $A, k_{B}, k_{C}, \alpha$ and $\tau$ are extracted from calibration data, consisting of gamma lines from deployed and naturally occurring radioisotopes ${ }^{68} \mathrm{Ge}(\gamma 1.02$ $\mathrm{MeV}),{ }^{60} \mathrm{Co}(\gamma 2.5 \mathrm{MeV})$ and ${ }^{241} \mathrm{Am}-{ }^{13} \mathrm{C}$ neutron source $(\gamma 8 \mathrm{MeV})$ as well as the continuous electron spectrum from decays of cosmogenic ${ }^{12} \mathrm{~B}$.

Corrections are done for calibration lines shift caused by Compton scattering of gamma's in the body of the calibration source and by shielding of the scintillations by the calibration- source body. Other corrections are done for scintillation-light collection non-uniformity in the detector, and stability. The precision of the model is better than $0.5 \%$ for prompt energy larger than $2 \mathrm{MeV}$.

\section{References}

[1] D. Adey et al., collaboration Daya Bay, Measurement of electron antineutrino oscillation with 1958 days of operation at Daya Bay, arXiv:1809.02261, submitted to Phys.Rev.Lett. 